
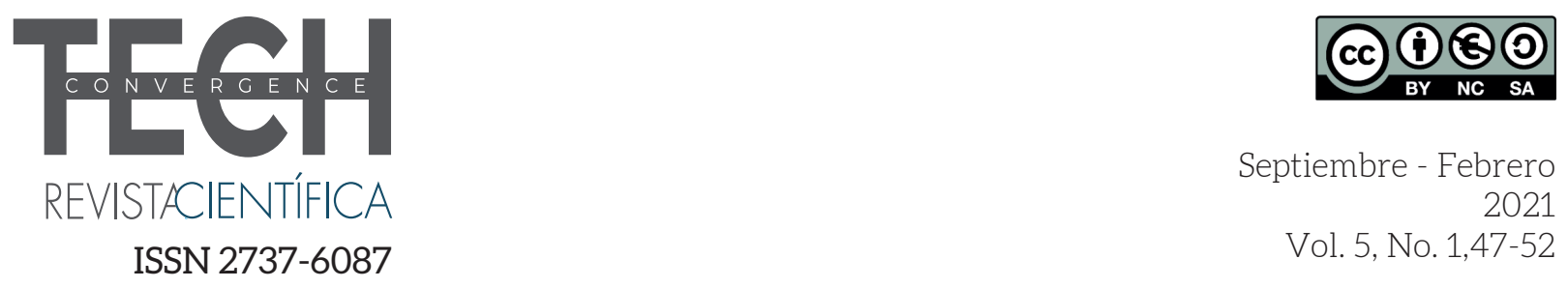

\title{
LA EDUCACIÓN 4.0 Y SU IMPACTO EN LA FORMACIÓN DOCENTE Y EN EL APRENDIZAJE DE LOS ALUMNOS
}

\section{Daniel Claudio Perazzo Logioia}

Vicerrector del Instituto Superior Tecnológico Particular Sudamericano, Cuenca E-mail: vicerectoradoits@sudamericano.edu.ec

(iD https://orcid.org/0000-0003-3089-2225
Fecha de recepción: 20/08/2020

Fecha de aceptación: 05/02/2021

\section{RESUMEN}

La educación 4.0 tiene que ver con generar una nueva revolución en los modelos educativos, ya que busca proporcionar a las nuevas generaciones la formación en las competencias que requiere para el siglo XXI, que además de la tecnología, tiene que ver con todo aquello que las máquinas no pueden hacer y en donde el ser humano marca una diferencia. Se presenta un estudio de tipo descriptivo-cuantitativo, que examina la aplicación de las TIC en los profesores pertenecientes a carreras del ámbito de las Ciencias Sociales en una universidad pública, con objetivo de identificar los recursos tecnológicos de educación 1.0 a la Educación 4.0 que utiliza el profesor en sus clases. Se aplicó un cuestionario a 38 profesores a docentes de diferentes disciplinas permitiendo determinar en qué grado se utilizan las tecnologías en clase, los objetivos que consideran los profesores a la hora de implementarlas y las actitudes que demuestran en relación a las TIC. Como afirma (Prats, 2016) "La cuestión no es estar a favor o en contra de la introducción de las tecnologías en la educación, ya que todos estamos a favor. Lo relevante es determinar qué papel deben jugar en un proceso de enseñanza aprendizaje". Para (Salvador, 1991) la computadora es un medio innovador, que provoca "...conseguir el aprendizaje como un material más dentro del aula" y que no sustituye al profesor, más bien viene a apoyar la labor del docente. En la actualidad es inexcusable que el docente reciba cursos y talleres que apoyen su labor cotidiana, con objeto de poder conocer y aplicar las TIC en consonancia con las exigencias del mundo laboral en el cual se desempeñará el alumno en un futuro próximo.

Palabras Clave: educación 4.0, aprendizaje, TICS.

\begin{abstract}
Education 4.0 has to do with generating a new revolution in educational models, since it seeks to provide new generations with the training in the skills required for the 21st century, which in addition to technology, has to do with everything that machines cannot do and where the human being makes a difference.
\end{abstract}

A descriptive-quantitative study is presented, which examines the application of ICT in professors belonging to careers in the field of Social Sciences in a public university, in order to identify the technological resources from Education 1.0 to Education 4.0 that it uses the teacher in his classes. A questionnaire was applied to 38 teachers to teachers of different disciplines, allowing to determine to what degree technolo- 
gies are used in class, the objectives that teachers consider when implementing them and the attitudes they show in relation to ICT. As stated (Prats, 2016) "The question is not to be in favor or against the introduction of technologies in education, since we are all in favor. The important thing is to determine what role they should play in a teaching-learning process ". For (Salvador, 1991) the computer is an innovative medium, which causes "... to get learning as one more material in the classroom" and which does not replace the teacher, rather it comes to support the work of the teacher. At present it is inexcusable that the teacher receives courses and workshops that support their daily work, in order to be able to know and apply ICT in line with the demands of the world of work in which they will work in the near future.

Keywords: education 4.0, learning, ICTS.

Forma sugerida de citar: Perazzo Logioia, D. C. (2021). La educación 4.0 y su impacto en la formación docente y en el aprendizaje de los alumnos. Convergence Tech Revista Científica. 5(1), 47-52. https://doi.org/10.53592/convtech. v5iV.3

\section{INTRODUCCIÓN}

$\mathbf{E}$ trabajo que presentamos tiene como referente una amplia investigación que indaga sobre los usos y actitudes de los profesores que pertenecen a diferentes facultades de una universidad pública mexicana. Este artículo está centrado en una de las facultades en donde se llevó a cabo el relevamiento de los datos, la Facultad de Ciencias Sociales. Pretendemos explicar y argumentar a través de la aplicación de una encuesta cómo los docentes propician el uso de la tecnología en clases, como así también los objetivos que consideran para su aplicación y las actitudes que despiertan en ellos la utilización de las TIC.

Los objetivos de este estudio son:

- Identificar los recursos tecnológicos que utiliza el profesor en sus clases.

- Apreciar la importancia que le brinda el docente a las TIC para fortalecer el proceso de enseñanza-aprendizaje de sus alumnos.

- Definir la categoría de objetivos que el docente considera para utilizar las TIC en la asignatura que imparte.

- Valorar las actitudes de los profesores en relación a las TIC.

Para que un profesor pueda encauzar a sus alumnos, primero debe estar preparado para ello, como dice (Perazzo, 1995) “En cierta medida, todos sabemos lo nece- sario de esta herramienta, en estos últimos años, en que toda la información se encuentra computarizada. Ejemplo de ello son los programas informáticos que nos presentan un gran paquete informativo: enciclopedias, por ejemplo, en entorno PC y bajo la modalidad de los sistemas multimedia... Las reformas educativas de los últimos años promueven, la implementación de las nuevas tecnologías. Es público que las reformas en un sistema educativo, tienen como objetivo prioritario mejorar la calidad de la enseñanza y adecuarla a las nuevas demandas sociales".

Bajo esta perspectiva consideramos que se hace obligatorio brindarles a los docentes un conocimiento que guíe sus decisiones curriculares y ofrezca sugerencias para la práctica educativa. Es urgente fortalecer la formación técnica pedagógica, la cual consistiría en una aproximación amplia a las posibilidades educativas que ofrecen las TIC, contextualizadas en distintos modelos de enseñanza y aprendizaje (Uzcategui y González, (2018). No dejemos de lado la formación instrumental, es decir, la familiarización con la computadora y todos los periféricos necesarios con los programas más adecuados para utilizar todo el software disponible de la forma más conveniente, sin que atente con la identidad profesional del profesor, ni le cree inseguridad. Es preciso promover una formación docente autorreflexiva a partir del análisis del rol del profesor, en un contexto en el cual los alumnos poseen amplios conocimientos de tecnología, muchas veces sin aplicarla al medio educativo y que pudieran favorecer su aprendizaje. Esto viene dado por la falta de experiencia y conocimiento en el docente para hacer uso adecuado de ella en la asignatura que imparte. 


\section{UTILIZACIÓN DE LAS TIC EN EL AULA}

Cada vez vemos mayor número de alumnos que saben acceder de forma rápida a grandes cantidades de información, ya que se encuentran acostumbrados a un lenguaje más global. El desarrollo tecnológico ha provocado el auge de nuevas habilidades en los estudiantes que deben ser tenidos en cuenta. Por lo tanto, los profesores deben asumir la utilización de las nuevas tecnologías en el trabajo sin dejar de mencionar que deberán estar apoyados por las autoridades educativas, tanto en su propia preparación profesional, como en la dotación de tecnologías educativas en el ámbito institucional.

La gran cantidad de información que se mueve en nuestro entorno ha dejado en manos de educadores una larga tradición centrada en la transmisión de información como herramienta de enseñanza-aprendizaje. En la actualidad, hay un mayor número de información que aparece en internet y en las redes, con lo cual los jóvenes de hoy tienen un acceso ilimitado a ella.

El uso de los medios informáticos en el proceso de enseñanza-aprendizaje no es nuevo, pero la frecuencia y tiempo con que los alumnos los usan están relacionados directamente con su edad. Actualmente la educación en los diferentes centros de estudios, sean de carácter infantil, de enseñanza media o superior, se encuentra encaminada hacia la competencia de "aprender a aprender", e intenta dejar a un lado la acumulación de información para acercarse al saber utilizar herramientas y recursos que permitan a los alumnos a aprender, concretar qué información se necesita en cada momento, cómo localizarla, recuperarla, procesarla, reelaborarla, cómo comunicar sus percepciones en torno a ella, extraer el conocimiento necesario en cada momento y para cada situación y construir el propio conocimiento sobre la base de una búsqueda activa.

Por lo tanto, como nos indican (Hernández, Acevedo, Martínez y Cruz, 2014) "El uso efectivo de las TIC en el aula depende de la efectividad y eficacia de los procesos de enseñanza-aprendizaje desarrollados y de la capacidad de los docentes y estudiantes para interactuar con los recursos tecnológicos".

\section{METODOLOGÍA}

Consideramos que la metodología más acorde al tipo de estudio que planteamos es la cuantitativa, con objeto de obtener información de primera mano de los profeso- res encuestados. Se llevó a cabo un análisis cruzado, "...lo cual implica una representación tabular de datos numéricos, por lo general representados en forma de frecuencia y porcentaje, en la cual las variables se dividen en forma cruzada para estudiar sus relaciones (Jiménez, 2021). Este análisis cruzado permite al investigador determinar la naturaleza de las relaciones entre las variables". (Ortiz, 2016, p.15) Para ello aplicamos un instrumento con 25 reactivos a 38 profesores de la Facultad de Ciencias Sociales de una universidad pública mexicana, los cuales forman parte de las carreras de Derecho (24) y Economía (14).

\section{RESULTADOS}

La formación del profesorado en TIC implica actividades que incidan en el "perfeccionamiento de la función docente" $y$, por lo tanto, en el aumento de la calidad de la enseñanza. Deberá gestionarse al interior de la institución una formación en TIC que permita desarrollar la competencia digital de manera continua, lo cual implica como lo refiere Marcelo (1989) "...como un proceso de desarrollo profesional en el que la formación inicial es la primera fase (interconexión entre la formación inicial y el currículum de la formación permanente) y la integración de contenidos propiamente académicos-disciplinares y la formación pedagógica de los profesores".

La decisión de cada profesor, por utilizar innovaciones tecnológicas, está asociada a sus propias perspectivas de valores (esfuerzo, tiempo, riesgo) y compensaciones de tipo profesional, ya sea simbólicas, no económicas o pedagógicas.

En un intento por resolver este dilema, podemos plantear la siguiente ecuación:

\section{V.I. $=C-(E+R)$}

\section{Dónde:}

V.I. = Valor de la innovación tecnológica a tener en cuenta en la decisión que adopte el profesor.

C = Compensaciones o recompensas que pueda obtener (simbólicas o pedagógicas).

$E=$ Los esfuerzos que conllevan al hecho de innovar tecnológicamente, o sea, el tiempo y la capacitación profesional.

$\boldsymbol{R}=$ Los riesgos que entraña la inclusión de la innovación tecnológica en el aula. 
Es decir que el valor de cualquier innovación, vendrá

y recompensas que el profesor pueda obtener y los esdeterminada por la diferencia entre las compensaciones

fuerzos y riesgos que tal innovación entrañe.

\begin{tabular}{|c|c|c|c|c|c|c|c|c|c|c|c|c|c|c|c|}
\hline \multirow{2}{*}{ Categoría } & \multicolumn{7}{|c|}{ Porcentaje } & \multicolumn{6}{|c|}{ Frecuencia } & \multirow{2}{*}{ Moda } & \multirow{2}{*}{ Media } \\
\hline & 0 & 1 & 2 & 3 & 3 & 4 & 5 & 0 & 1 & 2 & 3 & 4 & 5 & & \\
\hline Transparencias & 0,0 & 16,7 & 29, & 345 & 88 & 8,3 & 0,0 & 0 & 4 & 7 & 11 & 2 & 24 & 3 & 2,46 \\
\hline Diapositivas & 0,0 & 33,3 & 20,8 & $8 \quad 37$ & 1,58 & 8,3 & 0,0 & 0 & 8 & 5 & 9 & 2 & 0 & 3 & 2,21 \\
\hline Videogramas & 0,0 & 37,5 & 16, & $7 \quad 33$ & 3 & 8,3 & 4,2 & 0 & 9 & 4 & 8 & 2 & 1 & 1 & 2,25 \\
\hline Filmes/Video & 0,0 & 20,8 & 33, & 329 & 2 & 8,3 & 8,3 & 0 & 5 & 8 & 7 & 2 & 2 & 2 & 2,50 \\
\hline Televisión & 0,0 & 25,0 & 20,8 & 833 & 32 & 26,7 & 4,2 & 0 & 6 & 5 & 8 & 4 & 1 & 3 & 2,54 \\
\hline Videodiscos & 4,2 & 45,8 & 12,5 & $5 \quad 25$ & 0 & 8,3 & 4,2 & 1 & 11 & 3 & 6 & 2 & 1 & 1 & 2,00 \\
\hline Grabaciones sonoras & 0,0 & 50,0 & 8,3 & 25 & 1,0 & 12,5 & 4,2 & 0 & 12 & 2 & 6 & 3 & 1 & 1 & 2,13 \\
\hline Computadoras & 0,0 & 29,2 & 33, & 320 & $1,8 \quad 1$ & 12,5 & 4,2 & 0 & 7 & 8 & 5 & 3 & 1 & 2 & 2,29 \\
\hline $\begin{array}{c}\text { Materiales Hipermedia/ } \\
\text { Multimedia }\end{array}$ & 0,0 & 45,8 & 29,2 & 216 & 7 & 8,3 & 0,0 & 0 & 11 & 7 & 4 & 2 & 0 & 1 & 1,88 \\
\hline Radio & 0,0 & 62,5 & 25, & 0 & & 12,5 & 0,0 & 0 & 15 & 6 & 0 & 3 & 0 & 1 & 1,63 \\
\hline Datashow & 0 & 66,7 & 16, & 7 & & 8,3 & 0,0 & 0 & 16 & 4 & 2 & 2 & 0 & 1 & 1,58 \\
\hline Software tutorial & 0,0 & 70,8 & 8,3 & 16 & 7 & 4,2 & 0,0 & 0 & 17 & 2 & 4 & 1 & 0 & 1 & 1,54 \\
\hline Power Point & 0,0 & 33,3 & 25, & $0 \quad 25$ & 10 & 12,5 & 4,2 & 0 & 8 & 6 & 6 & 3 & 1 & 1 & 2,29 \\
\hline Software general & 0,0 & 29,2 & 12, & $5 \quad 25$ & $0 \quad 2$ & 29,2 & 4,2 & 0 & 7 & 3 & 6 & 7 & 1 & 2 & 2,67 \\
\hline Photoshop & 4,2 & 70,8 & 8,3 & 12 & 1.5 & 4,2 & 0,0 & 1 & 17 & 2 & 3 & 1 & 0 & 1 & 1,42 \\
\hline \multicolumn{16}{|c|}{ Tabla 2: Actitudes de los profesores en relación con las TIC (Carrera: Derecho) 24 encuestados } \\
\hline \multirow{2}{*}{\multicolumn{2}{|c|}{ Categoría }} & \multicolumn{6}{|c|}{ Porcentaje } & \multicolumn{6}{|c|}{ Frecuencia } & \multirow{2}{*}{ Moda } & \multirow{2}{*}{ Media } \\
\hline & & 0 & 1 & 2 & 3 & 4 & 5 & 0 & 1 & 2 & 3 & 4 & 5 & & \\
\hline \multicolumn{2}{|c|}{$\begin{array}{l}\text { La enseñanza es causa directa } \\
\text { del aprendizaje }\end{array}$} & 4,2 & 8,3 & 16,7 & 33,3 & 33,3 & 4,2 & 1 & 2 & 4 & 8 & 8 & 1 & 4 & 2,96 \\
\hline \multicolumn{2}{|c|}{$\begin{array}{l}\text { El aprendizaje se mide con } \\
\text { relación a reglas/patrones }\end{array}$} & 0,0 & 4,2 & 4,2 & 33,3 & 33,3 & 25,0 & 0 & 1 & 1 & 8 & 8 & 6 & 4 & 3,71 \\
\hline \multicolumn{2}{|c|}{$\begin{array}{l}\text { El aprendizaje es resultado de } \\
\text { un conjunto de procesos }\end{array}$} & 0,0 & 4,2 & 16,7 & 12,5 & 33,3 & 33,3 & 0 & 1 & 4 & 3 & 8 & 8 & 5 & 3,75 \\
\hline \multicolumn{2}{|l|}{$\begin{array}{c}\text { El aprendizaje se evalúa a } \\
\text { partir de la aplicación de } \\
\text { conocimientos }\end{array}$} & 0,0 & 0,0 & 12,5 & 25,0 & 54,2 & 8,3 & 0 & 0 & 3 & 6 & 13 & 2 & 4 & 3,58 \\
\hline \multicolumn{2}{|c|}{$\begin{array}{c}\text { Enseñar bien es lo bastante } \\
\text { para aprender bien }\end{array}$} & 0,0 & 4,2 & 4,2 & 33,3 & 33,3 & 25,0 & 0 & 1 & 1 & 8 & 8 & 6 & 4 & 3,71 \\
\hline \multicolumn{2}{|c|}{$\begin{array}{l}\text { Usar la técnica más apropiada } \\
\text { no es sinónimo de eficacia }\end{array}$} & 4,2 & 3,2 & 25,0 & 37,5 & 16,7 & 12,5 & 1 & 1 & 6 & 9 & 4 & 3 & 3 & 2,96 \\
\hline \multicolumn{2}{|l|}{$\begin{array}{c}\text { El profesor prepara } \\
\text { pormenorizadamente las } \\
\text { respuestas }\end{array}$} & 0,0 & 0,0 & 37,5 & 20,8 & 25,0 & 16,7 & 0 & 0 & 9 & 5 & 6 & 4 & 2 & 3,21 \\
\hline \multicolumn{2}{|l|}{$\begin{array}{c}\text { Contenidos más cercanos } \\
\text { aprendizaje más eficaz }\end{array}$} & 0,0 & 8,3 & 25,0 & 25,0 & 33,3 & 8,3 & 0 & 2 & 6 & 6 & 8 & 2 & 4 & 3,08 \\
\hline
\end{tabular}




\section{DISCUSIÓN}

\section{Necesidad de la formación del profesorado en las TIC}

Para (Pantoja, 1995) "La formación docente, es un proceso sistemático, abierto y permanente cuyo propósito, es el de formar a los profesores para que adquieran las competencias docentes y profesionales necesarias para el desarrollo de su práctica docente y profesional bajo los lineamientos teóricos y metodológicos de las actuales corrientes didácticas, sociales, psicológicas, que le posibiliten, el transformar su entorno social, político y económico". A la luz de los resultados obtenidos, en este caso la formación del profesorado en TIC implica actividades que incidan en el "perfeccionamiento de la función docente" $y$, por lo tanto, en el aumento de la calidad de la enseñanza. Deberá gestionarse al interior de la institución una formación en TIC que permita desarrollar la competencia digital de manera continua, lo cual implica como lo refiere (Marcelo, 1989) "...como un proceso de desarrollo profesional en el que la formación inicial es la primera fase (interconexión entre la formación inicial y el currículum de la formación permanente) y la integración de contenidos propiamente académicos-disciplinares y la formación pedagógica de los profesores".

La formación del profesorado adquiere un interés cada vez mayor y supone un desafío para el formador, hay interés por parte de la tecnología educativa en promover:

A. La formación de docentes para el uso de las TIC.

B. La formación de docentes con TIC.

C. El análisis de las actitudes de los profesores ante la tecnología educativa.

D. Los enfoques metodológicos para la preparación inicial y permanente de los profesores en las modalidades de la utilización de la tecnología educativa.

La metodología aplicada en la formación de los profesores, debe asumir el repertorio de técnicas que se desea que el alumno aplique en su futuro ejercicio profesional. Con lo cual la formación de profesores en TIC hace posible:
1. Una mejor estructura del contenido.

2. Estandarizar ciertas decisiones en materia educativa.

3. Hacer más formal la instrucción.

4. Rentabilizar el tiempo dedicado a la enseñanza.

5. Promover una enseñanza más interactiva.

6. Flexibilizar el tiempo dedicado a la enseñanza.

7. Cambiar el rol del profesor, de enseñante a facilitador.

Con la necesidad de implementar la tecnología en el aula, urge entonces la obligación de capacitar a los profesores en el dominio de las TIC, a través de una pedagogía de la imagen y del uso racional y crítico de los recursos tecnológicos en su aplicación a la educación. He ahí los retos a los que se tienen que afrontar las instituciones educativas en la formación de los docentes, por lo tanto, será preciso contar con un elevado número de profesores en los ámbitos de formación.

Hemos de entender, y así lo confirman algunos estudios realizados por (Grau, 1996) que la decisión de cada profesor, por utilizar innovaciones tecnológicas, está asociada a sus propias perspectivas de valores (esfuerzo, tiempo, riesgo) y compensaciones de tipo profesional, ya sea simbólicas, no económicas o pedagógicas, mejorar su imagen como docente, mejorar la relación con los alumnos, beneficio del aprendizaje de los alumnos, entre otras.

En un intento por resolver este dilema, podemos plantear la siguiente ecuación:

\section{V.I. $=C-(E+R)$}

\section{Dónde:}

V.I. = Valor de la innovación tecnológica a tener en cuenta en la decisión que adopte el profesor.

$\boldsymbol{C}=$ Compensaciones o recompensas que pueda obtener (simbólicas o pedagógicas). 
$\boldsymbol{E}=$ Los esfuerzos que conlleva el hecho de innovar tecnológicamente, o sea, el tiempo y la capacitación profesional.

$\boldsymbol{R}=$ Los riesgos que entraña la inclusión de la innovación tecnológica en el aula.

Es decir que el valor de cualquier innovación, vendrá determinada por la diferencia entre las compensaciones y recompensas que el profesor pueda obtener y los esfuerzos y riesgos que tal innovación entrañe.

Como afirma (López, 2017, p. 301) "Sin duda el uso que las TIC ha tenido en la educación está estrechamente relacionado con la mente de los usuarios. En principio a estas herramientas se les vio como máquinas para procesar datos; posteriormente se les asoció con el uso de programas de propósito general o de productividad. Más adelante permitió el desarrollo de los programas multimedia. En las décadas de 1980 y 1990 a las TIC se les pudo ver como auténticas herramientas cognitivas. En la actualidad, y con el uso de internet, las TIC ayudan a conformar complejas comunidades virtuales en las que sobresale el desarrollo de una inteligencia colectiva, sumado el talento y el potencial de muchos, en una sola aplicación".

\section{CONCLUSIONES}

La necesidad de formación del profesorado a través de las TIC para su aplicación en el aula se avanzará a medida que se haga extensiva su labor en la institución educativa, mediante la formación a través de formadores que reproduzcan y adapten las experiencias de formación en este aspecto a su trabajo en el aula.

Es importante se forme un educador idóneo con vocación por el hombre, que esté capacitado para trabajar en diferentes realidades de vida, en otras palabras, un profesional de la educación efectivo y pertinente.

\section{BIBLIOGRAFÍA}

Grau, J. (1996). La Informática Educativa en el Marco de la Educación. Escuela Universitaria de Magisterio de Toledo; XXI.

Hernández, L.; Acevedo, J.; Martínez, C. \& Cruz, B. (2014). El uso de las TIC en el aula: un análisis en términos de efectividad y eficacia. Congreso Iberoamericano de Ciencia, Tecnología, Innovación y Educación; 1-21.

Jiménez González, L. L. (2021). Impacto de la investigación cuantitativa en la actualidad. Convergence Tech, 4(1), 59-68. Recuperado a partir de http://revista.sudamericano.edu.ec/index.php/convergence/article/view/35

López, M. (2017) Aprendizaje, competencias y TIC. Pearson: México.

Marcelo, C. (1989). Introducción a la formación del profesorado. Universidad de Sevilla: Sevilla, España.

Ortiz, F. (2016) Diccionario de Metodología de la Investigación Científica. Limusa: México.

Pantoja Sánchez, M. (1995). Propuesta de un Diplomado en Docencia Universitaria a Distancia para Profesores de las Facultades de Contaduría y Administración en las Universidades del Interior de la República Mexicana. Tesis de Maestría en Tecnología de la Educación. Universidad de Salamanca: Salamanca, España.

Prats, J. (2016). Internet en las aulas. Mamakuna; 2; 60-66. Universidad Nacional de Educación: Ecuador.

Perazzo, D. (1995). Propuesta de un Modelo Explicativo para la Innovación en los Centros Educativos. I Congreso de Innovación Educativa; I; 365-370. Universidad de Santiago de Compostela: Galicia, España.

Perazzo, D. (1996). La Innovación en los Centros Educativos. Actas del V Congreso del MERCOSUR. Buenos Aires, Argentina.

Salvador, A. (1991). La Informática en la Acción Educativa. M.E.C. Madrid, España.

Solomón, J. (1987). Entorno de Aprendizajes con Ordenadores. Una Reflexión sobre las Teorías del Aprendizaje y la Educación. Paidós: Madrid.

Uzcategui, Y. J. A. H., \& González, L. L. J. (2018). Propuesta metodológica para la orientación de la función docente en el contexto de municipalización de la educación universitaria. Conocimiento Libre y Licenciamiento (CLIC), (17). https://convite.cenditel.gob.ve/revistaclic/index.php/revistaclic/article/view/920 